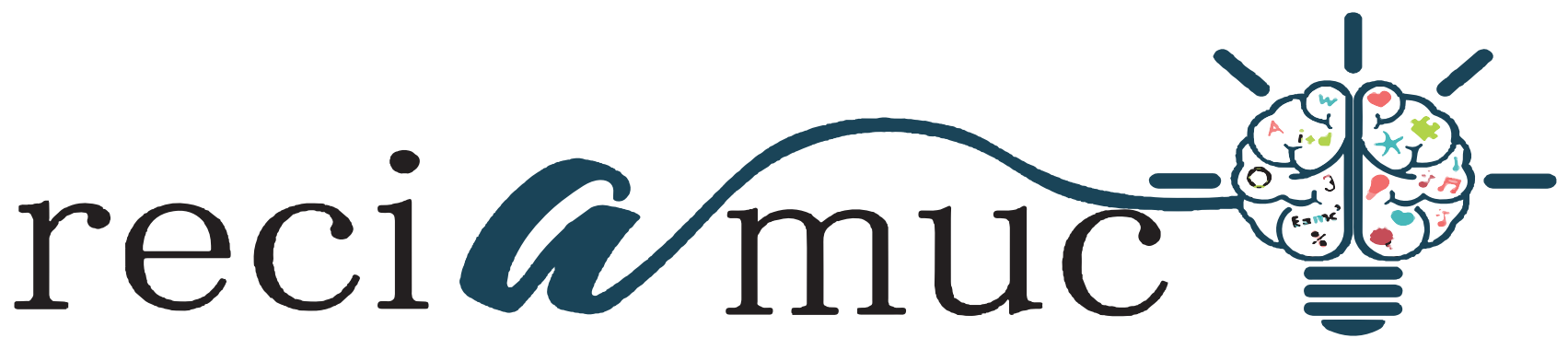

DOI: 10.26820/reciamuc/5.(4).noviembre.2021.92-99

URL: https://reciamuc.com/index.php/RECIAMUC/article/view/730

EDITORIAL: Saberes del Conocimiento

REVISTA: RECIAMUC

ISSN: 2588-0748

TIPO DE INVESTIGACIÓN: Artículo de Revisión

CóDIGO UNESCO: 32 Ciencias Médicas

PAGINAS: 92-99

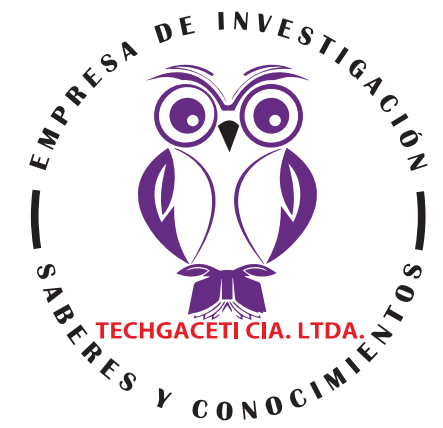

\title{
Pioderma gangrenoso
}

Pyoderma gangrenosum

Pioderma gangrenoso

\section{Karen Michelle Ruilova Gavilanes'; Ari Estefano Jerves Crespo²; Luis Andrés Bodero Acuña3; Ana Gabriela Carrera Chulde ${ }^{4}$}

RECIBIDO: 15/09/2021 ACEPTADO: 05/10/2021 PUBLICADO: 29/11/2021

1. Médica; Investigador Independiente; Quito, Ecuador; krenmr@gmail.com; (D) https://orcid.org/0000-00015689-5067

2. Médico; Investigador Independiente; Cuenca, Ecuador; ajerves22@gmail.com; (D) https://orcid.org/0000-00022125-1656

3. Magister en Seguridad y Salud Ocupacional; Médico Cirujano; Investigador Independiente; Quito, Ecuador; luisandresbd@gmail.com; (D) https://orcid.org/0000-0002-6387-7251

4. Médica; Investigador Independiente; Quito, Ecuador; agcarrera_4@hotmail.com; (iD https://orcid.org/00000003-0980-6054

\section{CORRESPONDENCIA}

Karen Michelle Ruilova Gavilanes

krenmr@gmail.com

\section{Quito, Ecuador}

@ RECIAMUC; Editorial Saberes del Conocimiento, 2021 


\section{RESUMEN}

El pioderma gangrenoso (PG) es una dermatosis neutrofílica infrecuente, crónica, dolorosa y recidivante, idiopática en el 25-50\% de los pacientes, con una presentación morfológica distintiva pero variable que abarca las formas pustulosas, ampollosa, ulcerativa y granulomatosa superficial o vegetante. De forma característica, en el $20-50 \%$ de las ocasiones aparece sobre piel previamente traumatizada; hasta en un $75 \%$ de los casos se asocia a una enfermedad sistémica previa, concurrente o posterior a su aparición, como la enfermedad inflamatoria intestinal (colitis ulcerativa, enfermedad de Crohn, 20-30\%), poliartritis (20\%), trastornos hematológicos (leucemia mieloide aguda y crónica, tricoleucemia, mielodisplasia, gammapatía monoclonal lgA) (15-25\%), puede ser una manifestación cutánea de enfermedades autoinflamatorias monogénicas, como el síndrome PAPA (pyogenic arthritis, PG and acne), PASH (PG, acne and suppurative hidradenitis) o PAPASH (pyogenic arthritis, acne, PG and suppurative hidradenitis) o aparecer como acompañante de una dermatosis pustulosa subcórnea, enfermedad de Behçet o del síndrome de Sweet; raramente se debe a medicamentos. La metodología utilizada para el presente trabajo de investigación, se enfoca hacia una metodología orientada hacia la necesidad de indagar en forma precisa y coherente una situación. Enmarcada dentro de una revisión bibliográfica de tipo documental, ya que nos vamos a ocupar de temas planteados a nivel teórico como es Pioderma gangrenoso. La técnica para la recolección de datos está constituida por materiales impresos, audiovisuales y electrónicos, estos últimos como Google Académico, PubMed, entre otros. La información aquí obtenida será revisada para su posterior análisis. En base a los casos aquí presentados, se confirma lo analizado en la literatura revisada, que los casos de piodermas gangrenosos, se controlan con farmacología, específicamente con corticoesteroides, analgésicos y desinflamatorios, que en la mayoría de los casos evolucionan favorablemente. Otro punto a considerar es que también se prueba que otras patologías o comorbilidades que pueda tener un paciente, pueden desencadenar procedimientos que terminen en piodermas gangrenosos, en uno de los casos clínicos aquí presentados uno de los pacientes presento lesión ulcerada probablemente como consecuencia de la diabetes mellitus que padece desde hace mucho tiempo, en otros casos es posible la generación de esta patología como enfermedades de Crohn, leucemia, artritis reumatoide, entre otras.

Palabras clave: Gangrenoso, Corticoesteroides, Lesión, Ulcera, Idiopática.

\section{ABSTRACT}

Pyoderma gangrenosum (PG) is a rare, chronic, painful and recurrent neutrophilic dermatosis, idiopathic in $25-50 \%$ of patients, with a distinctive but variable morphological presentation that includes pustular, bullous, ulcerative and superficial or vegetating granulomatous forms. Characteristically, in $20-50 \%$ of the cases it appears on previously traumatized skin; In up to $75 \%$ of cases it is associated with a previous, concurrent or subsequent systemic disease, such as inflammatory bowel disease (ulcerative colitis, Crohn's disease, 20-30\%), polyarthritis (20\%), hematological disorders (acute and chronic myeloid leukemia, hairy cell leukemia, myelodysplasia, monoclonal IgA gammopathy) (15-25\%), can be a cutaneous manifestation of monogenic autoinflammatory diseases, such as PAPA syndrome (pyogenic arthritis, PG and acne), PASH (PG, acne and suppurative hidradenitis) or PAPASH (pyogenic arthritis, acne, PG and suppurative hidradenitis) or appear as a companion to a subcorneal pustular dermatosis, Behcet's disease or Sweet's syndrome; it is rarely due to medications. The methodology used for this research work is focused on a methodology oriented towards the need to investigate a situation precisely and coherently. Framed within a bibliographic review of a documentary type, since we are going to deal with issues raised at a theoretical level such as Pyoderma gangrenosum. The technique for data collection is made up of printed, audiovisual and electronic materials, the latter such as Google Scholar, PubMed, among others. The information obtained here will be reviewed for further analysis. Based on the cases presented here, the analysis in the reviewed literature confirms that cases of gangrenous pyoderma are controlled with pharmacology, specifically with corticosteroids, analgesics and anti-inflammatory drugs, which in most cases evolve favorably. Another point to consider is that it is also proven that other pathologies or comorbidities that a patient may have, can trigger procedures that end in gangrenous pyoderma.In one of the clinical cases presented here, one of the patients presented an ulcerated lesion, probably as a consequence of diabetes. mellitus that you have suffered for a long time, in other cases it is possible to generate this pathology such as Crohn's diseases, leukemia, rheumatoid arthritis, among others.

Keywords: Gangrenous, Corticosteroids, Lesion, Ulcer, Idiopathic.

\section{RESUMO}

O pioderma gangrenoso (PG) é uma dermatose neutrofílica rara, crônica, dolorosa e recorrente, idiopática em 25-50\% dos pacientes, com apresentação morfológica distinta, mas variável, que inclui as formas pustulosa, bolhosa, ulcerativa e granulomatosa superficial ou vegetante. Caracteristicamente, em 20-50\% dos casos aparece em pele previamente traumatizada; Em até $75 \%$ dos casos, está associada a uma doença sistêmica anterior, concomitante ou subsequente, como doença inflamatória intestinal (colite ulcerosa, doença de Crohn, 20-30\%), poliartrite (20\%), distúrbios hematológicos (agudos e crônicos leucemia mieloide, leucemia de células pilosas, mielodisplasia, gamopatia IgA monoclonal) (15-25\%), pode ser uma manifestação cutânea de doenças autoinflamatórias monogênicas, como a síndrome PAPA (artrite piogênica, PG e acne), PASH (PG, acne e supurativa hidradenite) ou PAPASH (artrite piogênica, acne, PG e hidradenite supurativa) ou aparecem como acompanhantes de dermatose pustulosa subcórnea, doença de Behçet ou síndrome de Sweet; raramente é devido a medicamentos. A metodologia utilizada para este trabalho de investigação centra-se numa metodologia orientada para a necessidade de investigar uma situação de forma precisa e coerente. Enquadrado dentro de uma revisão bibliográfica de tipo documental, uma vez que trataremos de questões levantadas em um nível teórico como o Pioderma gangrenoso. A técnica de coleta de dados é composta por materiais impressos, audiovisuais e eletrônicos, estes últimos como Google Scholar, PubMed, entre outros. As informações aqui obtidas serão revisadas para análises posteriores. Com base nos casos aqui apresentados, a análise da literatura revisada confirma que os casos de piodermite gangrenosa são controlados com farmacologia, especificamente com corticosteroides, analgésicos e antiinflamatórios, que na maioria dos casos evoluem favoravelmente. Outro ponto a se considerar é que também está comprovado que outras patologias ou comorbidades que um paciente possa apresentar podem desencadear procedimentos que terminam em pioderma gangrenosa. Em um dos casos clínicos aqui apresentados, um dos pacientes apresentava lesão ulcerada, provavelmente como uma conseqüência do diabetes. mellitus que você sofre há muito tempo, em outros casos é possível gerar essa patologia como doenças de Crohn, leucemia, artrite reumatóide, entre outras.

Palavras-chave: Gangrenoso, Corticosteróides, Lesão, Úlcera Idiopática. 


\section{Introducción}

El pioderma gangrenoso (PG) es una enfermedad inflamatoria de la piel que fue descrita por primera vez por Brunsting y colaboradores en 1930: presentaron cinco pacientes con erupciones cutáneas, cuatro presentaban colitis ulcerosa. Lo denominó como pioderma gangrenoso debido a que pensó que se trataba de una infección estreptocócica que desarrollaba una gangrena cutánea. El término pioderma es considerado, en la actualidad, como equívoco porque la infección bacteriana como causa etiológica ha sido descartada, solo se mantiene por razones históricas (Pozo, Fleites, \& Pérez, 2021, págs. 612-613).

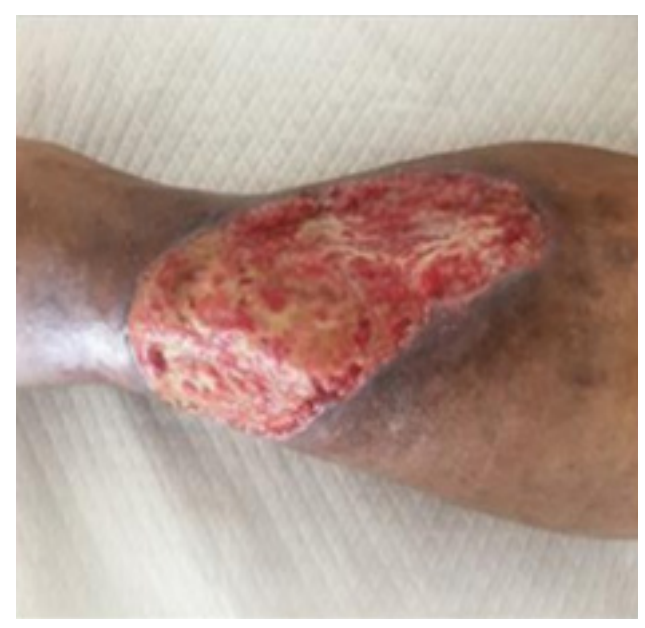

Figura 1. Ulcera de bordes irregulares.

Fuente: (Medina, Recalde, Vélez, Bravo, \& Sánchez, 2020)

El pioderma gangrenoso (PG) es una dermatosis neutrofílica infrecuente, crónica, dolorosa y recidivante, idiopática en el 25$50 \%$ de los pacientes, con una presentación morfológica distintiva pero variable que abarca las formas pustulosas, ampollosa, ulcerativa y granulomatosa superficial o vegetante. De forma característica, en el $20-50 \%$ de las ocasiones aparece sobre piel previamente traumatizada; hasta en un $75 \%$ de los casos se asocia a una enfermedad sistémica previa, concurrente o posterior a su aparición, como la en- fermedad inflamatoria intestinal (colitis ulcerativa, enfermedad de Crohn, 20-30\%), poliartritis (20\%), trastornos hematológicos (leucemia mieloide aguda y crónica, tricoleucemia, mielodisplasia, gammapatía monoclonal IgA) (15-25\%), puede ser una manifestación cutánea de enfermedades autoinflamatorias monogénicas, como el síndrome PAPA (pyogenic arthritis, PG and acne), PASH (PG, acne and suppurative hidradenitis) o PAPASH (pyogenic arthritis, acne, PG and suppurative hidradenitis) o aparecer como acompañante de una dermatosis pustulosa subcórnea, enfermedad de Behçet o del síndrome de Sweet; raramente se debe a medicamentos (Gaviria Castellanos, y otros, 2020).

Se caracteriza por presentar como lesión inicial un nódulo doloroso profundo o una pústula hemorrágica superficial de novo o posterior a un traumatismo mínimo, que se rompe y ulcera, eliminando exudado purulento y hemorrágico. Esto genera una úlcera con borde inflamatorio sobreelevado, de base necrótica rezumante. Es húmeda, horadada, perforada: a la compresión libera pus. El borde es irregular, dentado, rojo oscuro o púrpura, crece constantemente, rodeado por halo eritematoso brillante. Su base está cubierta parcialmente por material necrótico y ocupado por pequeños abscesos (Scasso, y otros, 2010). Se clasifica en cuatro variantes clínicas: ampollar, vegetativa, pustular y ulcerativa siendo esta última la forma más común. Las extremidades inferiores son las más frecuentemente implicadas, aunque otras áreas del cuerpo, así como membranas mucosas pueden estar involucradas (Medina, Recalde, Vélez, Bravo, \& Sánchez, 2020, pág. 55). 
Tabla 1. Enfermedades sistémicas asociadas con pioderma gangrenoso.

FRECUENTES

- Enfermedad deCrohn

- Colitis ulcerosa (trastorno dermatológico mas frecuente en esta enfermedad junto con el eritema nodoso)

- Artritis sero negativa

- Artitis reumatoidea

- Espondilitis

- Leucemia mieloide leucemia mieloide

- Gammapatía monoclonal lgA

- Enfermedad deTakayasu
RARAS

- Síndrome PAPA (acne quístico +artritis piógena +pioderma gangrenoso)

- Neumonitis, absceso pulmonar. Diabetes

- Síndrome SAPHO (sinovitis +acne +pustulosis +hiperostosis + osteítis)

- Gamapatía monoclonal

- Mieloma múltiple

- Mielodisplasia, policitemia vera, mielofibrosis

- Enfermedad de Hodking. Anemia hemolítica autoinmune

- Enfermedad de Behcet. Vasculitis necrosante

- Granulomatosis deWegener

- Lupus eritematoso sistémico. Anticuerpo antifosfolípido

- Leishmaniasis

- Cirrosis biliar primaria. Diverticulitis

- Otras dermatosis neutrofílicas

Fuente: (Scasso, y otros, 2010)

El diagnóstico de esta condición representa un reto, ya que suele manifestarse clínicamente con úlceras cutáneas, las cuales son comunes a muchas otras entidades de diferentes etiologías como la enfermedad vascular periférica, tumores, infecciones, vasculopatías y diferentes enfermedades inflamatorias La utilidad de la biopsia de piel es controvertida; se considera que puede realizarse para excluir otras posibles causas de las lesiones, pero no para establecer un diagnóstico preciso. Por lo anterior, es fundamental analizar los aspectos clínicos de los pacientes y correlacionarlos con los hallazgos histológicos, buscando características o asociaciones que permitan lograr un enfoque diagnóstico adecuado (Cuéllar, Zúñiga, Mejía, \& Londoño, 2019).

Aunque no existe un consenso de parámetros de laboratorio que confirmen el diagnóstico, se ha observado que se elevan los índices de eritrosedimentación, proteína C- reactiva, leucocitosis, puede coexistir anemia, no se observan anticuerpos específicos, aunque en algunos casos se ha observado títulos elevados de anticuerpos antinucleares (ANA) o anticuerpos anticitoplasma de neutrófilos (ANCA) (Medina,
Recalde, Vélez, Bravo, \& Sánchez, 2020, pág. 55). Debe solicitarse, además, cultivo de las lesiones para gérmenes comunes, micobacterias, hongos y leishmania con el fin de descartar etiología infecciosa, así como también estudios orientados hacia una patología vascular. Su manejo requiere habitualmente tratamiento local y sistémico (Lois, Pizzariello, Olivares, \& Maronna, 2011, pág. 25).

El tratamiento debe realizarse al pioderma gangrenoso y al trastorno sistémico asociado si lo hubiera. En cuanto a la evolución y pronóstico, tiene un curso impredecible: cura solo, permanece latente, o tiene una evolución crónica. Hay descritos diversos tratamientos locales y sistémicos, con resultados variables (Wong, Castellón, \& Rodríguez, 2019, pág. 2). La inmunosupresión es el pilar del tratamiento. Los medicamentos más comúnmente utilizados y con mejor experiencia clínica son los corticosteroides, la ciclosporina y los anticuerpos antifactor de necrosis tumoral alfa. Éstos últimos debido a su elevado coste no se encuentran disponibles de forma universal en la mayoría de los centros de salud. En este sentido, es fundamental contar con alternativas al tratamiento con- 
vencional en pacientes con PG y EC, y de esta forma iniciar un tratamiento temprano, efectivo y seguro en casos donde exista baja disponibilidad de las terapias convencionales. EI MTX es un fármaco análogo de ácido fólico, con actividad antiinflamatoria y antineoplásicas inhibiéndola enzima dihidrofolato reductasa. Una dosis $25 \mathrm{mg}$ subcutánea (SC) cada semana durante 12 a 16 semanas junto a dosis descendente de corticoides ha mostrado ser efectiva en la inducción de la remisión en pacientes con EC. No obstante, su eficacia y perfil de seguridad en el manejo de pacientes con PG y EC es incierta (Duarte-Chang \& Visuetti, 2019, págs. 175-176).

\section{Metodología}

La metodología utilizada para el presente trabajo de investigación, se enfoca hacia una metodología orientada hacia la necesidad de indagar en forma precisa y coherente una situación. Enmarcada dentro de una revisión bibliográfica de tipo documental, ya que nos vamos a ocupar de temas planteados a nivel teórico como es Pioderma gangrenoso. La técnica para la recolección de datos está constituida por materiales impresos, audiovisuales y electrónicos, estos últimos como Google Académico, PubMed, entre otros. La información aquí obtenida será revisada para su posterior análisis.

\section{Resultados}

\section{Caso 1:}

Duarte-Chang \& Visuetti (2019), presentan el caso de un varón de 39 años que acude al hospital con cuadro de diarrea crónica, dolor abdominal difuso, pérdida de peso, y fiebre acompañado de la aparición reciente de un nódulo, de unos $5 \mathrm{~cm}$, doloroso, eritematoso, con la presencia de una ampolla central.

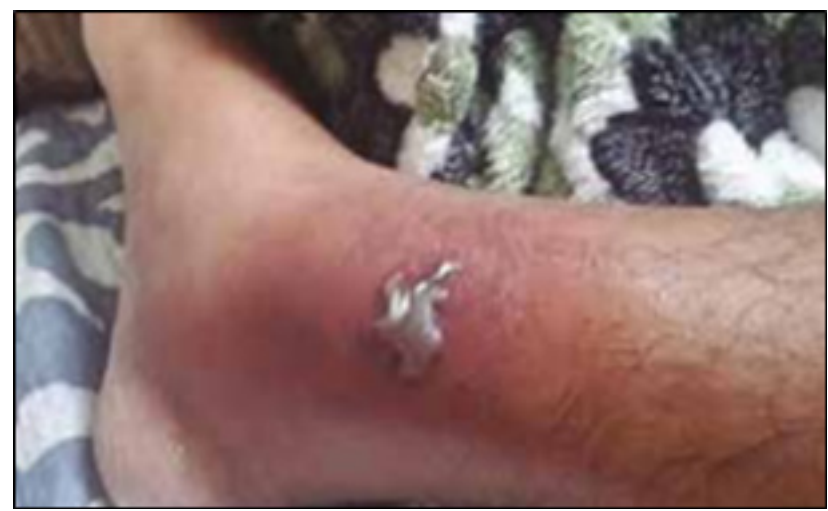

Figura 2. Nódulo doloroso, caliente al tacto, eritematoso, con ampolla central friable, situado en tercio distal de miembro inferior derecho.

Fuente: (Duarte-Chang \& Visuetti, 2019)

Se le realizó colonoscopia que mostró una mucosa eritematosa de aspecto empedrada, con la presencia de múltiples ulceras y exudados, con una distribución parcheada, con pérdida del patrón vascular normal y que respetaba el recto. Las biopsias reportaron hallazgos compatibles con EC. Se inició terapia con corticoides sistémicos con mejoría clínica del cuadro diarreico, sin embargo, la evolución de la ulcera pretibial no fue favorable. Por el contrario, aumentó de diámetro, con ruptura de la misma, eliminando un exudado purulento y hemorrágico resultando en una ulcera de bordes eritematosos y violáceos, infructuosos, socavados, con fondo rojo necrótico (Figura 2). La biopsia mostró hallazgos compatibles con PG y. De tal forma, se añadió la terapia con MTX, 25 mg SC cada semana con respuesta satisfactoria luego de 4 semanas y luego 16 semanas de tratamiento. A unas 24 semanas del diagnóstico de PG y de EC, cursa asintomático, sin brotes, sin recidivas, con la enfermedad en fase quiescente y evidente mejoría del PG. Su tratamiento de mantenimiento consiste en MTX con dosis de 15 mg cada semana asociado a prednisona $5 \mathrm{mg}$ vía oral cada día. En su seguimiento, no ha presentado efectos secundarios (pág. 176). 


\section{Caso II:}

Pozo, Fleites, \& Pérez (2021), presentan caso de paciente femenina, de la raza blanca, de 38 años de edad, que acudió a centro de salud, por presentar dolor a nivel del miembro inferior derecho de un mes de evolución. Al interrogatorio refirió que desde hacía un mes comenzó a presentar una lesión a nivel de la pierna derecha, como la "picada de un mosquito", que aumentaba de tamaño y se acompañaba de enrojecimiento y dolor; en esa ocasión acudió al médico, que hizo diagnóstico de linfangitis y que le indicó fomentos, de forma tópica, y ciprofloxacina como tratamiento sistémico, regresa a centro de salud ya que no mejora y aumento en la intensidad del dolor. Debido a perdida de piel en sitio de la lesión, se le realiza injerto de piel, luego de resultar negativos exámenes microbiológicos en el ara de cultivo, luego de realizarle el injerto de piel días después regresa a centro de salud por presentar abundante secreción serosanguinolenta y purulenta, y lesión ulceronecrótica que rodeaba el área del injerto. Ante la sospecha de PG se indicaron una química sanguínea y hemograma, un cultivo de secreción y una biopsia de piel y se comenzó tratamiento con prednisona (5mg), 40mg diarios, y salazosulfipiridina (500mg), $2 \mathrm{~g}$ diarios.

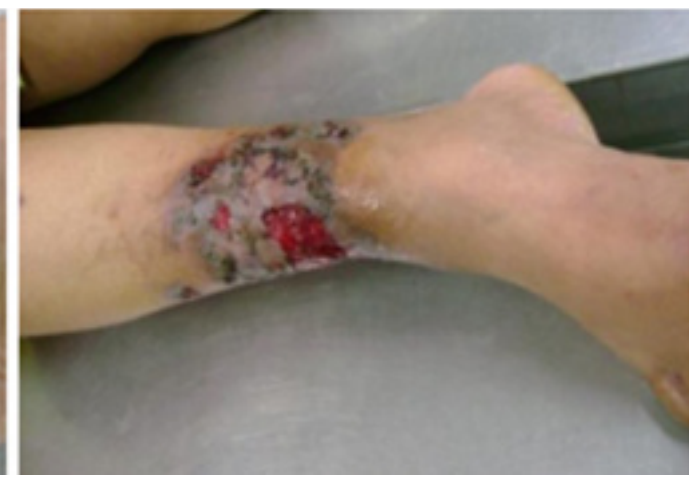

Figura 3. Lesión ulceronecrótica que rodea el área del injerto con abundante secreción serosanguinolenta y purulenta.

Fuente: (Pozo, Fleites, \& Pérez, 2021)

Comenzado el tratamiento la enferma refirió disminución del dolor en la zona y se observó una ostensible mejoría clínica, con ligera atrofia del área afectada. La paciente tiene seguimiento en la Consulta de Dermatología y no ha tenido remisión de los síntomas durante un año (págs. 613-615).

\section{Caso III:}

Wong, Castellón, \& Rodríguez (2019), presenta caso de paciente masculino de 73 años de edad, con antecedentes de diabetes mellitus tipo 2 desde hace 10 años, que se controla con dieta y glibenclamida (1 tableta diaria); hipertensión arterial hace 18 años, para lo tiene indicado tratamien- to con enalapril- $20 \mathrm{mg}$ (1 tableta diaria) y amlodipino $10 \mathrm{mg}$ (1 tableta diaria); y cardiopatía isquémica, para lo cual toma nitropental- 20 mg (2 tabletas diaria y aspirina $125 \mathrm{mg}$ diario). Acude a consulta por presentar lesión ulcerada en el dorso de pierna izquierda, en su tercio distal, acompañada de dolor y sensación de quemazón, con 8 meses de evolución. Por estos motivos se decide su ingreso para su mejor estudio y tratamiento.

Examen físico: Mucosas húmedas y normocoloreadas. En la piel presenta lesión ulcerada en pierna izquierda en su tercio distal, de forma ovalada, que mide aproximadamente $6 \mathrm{~cm} \times 4 \mathrm{~cm}$ de diámetro, de 
bordes bien delimitados, necróticos, violáceos y socavados, de base vegetante y superficie exudativa, acompañado de dolor intenso.

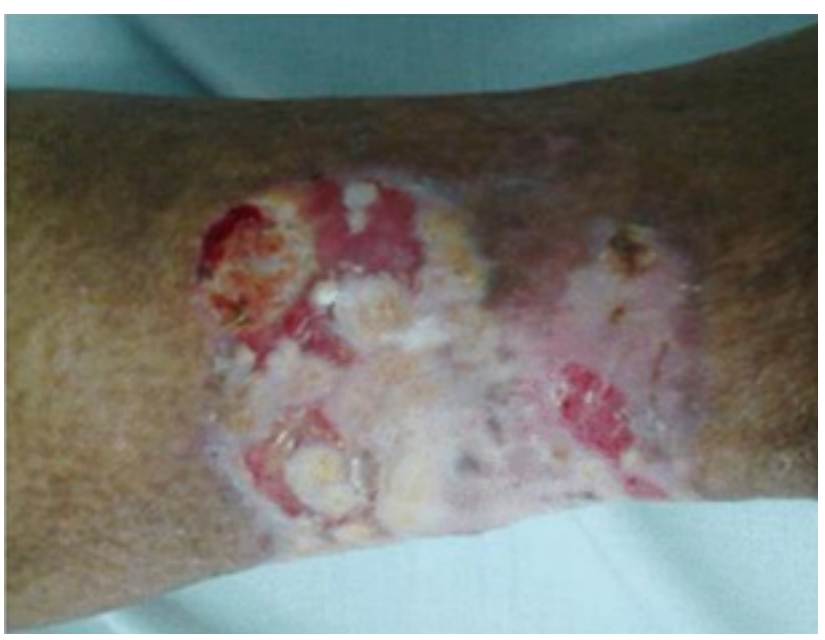

Figura 4. Lesión ulcerada en tercio distal de pierna izquierda

Fuente: (Wong, Castellón, \& Rodríguez, 2019)

Se concluye el caso como un pioderma gangrenoso y se inicia tratamiento con prednisona $80 \mathrm{mg}$ diarios durante 15 días. Esta dosis se disminuye progresivamente y en un plazo de 45 días se suspende el esteroide por vía sistémica. Se realiza además infiltración intralesional con acetato de triamcinolona 2 cc cada 21 días hasta la curación de la lesión, y se le indica al paciente Dapsone (100 mg) 1 tab diaria durante 3 meses. El paciente tuvo una evolución satisfactoria de la lesión (págs. 2-4).

\section{Conclusión}

En base a los casos aquí presentados, se confirma lo analizado en la literatura revisada, que los casos de piodermas gangrenosos, se controlan con farmacología, específicamente con corticoesteroides, analgésicos y desinflamatorios, que en la mayoría de los casos evolucionan favorablemente. Otro punto a considerar es que también se prueba que otras patologías o comorbilidades que pueda tener un paciente, pueden desencadenar procedi- mientos que terminen en piodermas gangrenosos, en uno de los casos clínicos aquí presentados uno de los pacientes presento lesión ulcerada probablemente como consecuencia de la diabetes mellitus que padece desde hace mucho tiempo, en otros casos es posible la generación de esta patología como enfermedades de Crohn, leucemia, artritis reumatoide, entre otras.

\section{Bibliografía}

Cuéllar, M. C., Zúñiga, E., Mejía, A., \& Londoño, A. (2019). Características clínicas e histopatológicas de pioderma gangrenoso en dos hospitales de Medellín, Colombia. Serie de casos. Medicina Cutánea Ibero-Latino-Americana, 46(3), 180-185.

Duarte-Chang, C., \& Visuetti, S. (2019). Tratamiento exitoso del pioderma grangrenoso con metotrexate en paciente con enfermedad de Crohn. Revista de Gastroenterología del Perú, 39(2), 175-177.

Gaviria Castellanos, J. L., Ávila León, J., Lara García, L., Bonilla Hernández, J., Motta Beltrán, A., \& Navarrete Aldana, N. (2020). Pioderma gangrenoso asociado a quemaduras: reporte de dos casos. Cirugía Plástica Ibero-Latinoamericana, 46(1), 79-84. doi:http://dx.doi.org/10.4321/S037678922020000100011

Lois, M., Pizzariello, G., Olivares, L., \& Maronna, E. (2011). Estudio retrospectivo de pacientes con pioderma gangrenoso en un período de 20 años y revisión de laliteratura. Dermatología Argentina, 18(2), 24-29.

Medina, D. T., Recalde, J., Vélez, V., Bravo, V., \& Sánchez, R. (2020). Pioderma Gangrenoso. Mediciencias UTA, 4(2), 54-59. doi:https://doi.org/10.31243/ mdc.uta.v4i2.349.2020

Pozo, Y. M., Fleites, D., \& Pérez, A. (2021). Pioderma gangrenoso. Informe de caso. Acta Médica del Centro, 15(4).

Scasso, M. S., Asensio, P., Pavón, G., Rico, M., Brusco, J., \& Martinez, P. (2010). Pioderma gangrenoso. Rev Hosp Privado Comunidad, 13(1), 36-9.

Wong, E. R., Castellón, D., \& Rodríguez, R. (2019). A propósito del pioderma gangrenoso. Folia Dermatológica Cubana, 10(3). 


\section{CITAR ESTE ARTICULO:}

Ruilova Gavilanes, K. M., Jerves Crespo, A. E., Bodero Acuña, L. A., \& Carrera Chulde, A. G. (2021). Pioderma gangrenoso. RECIAMUC, 5(4), 92-99. https:// doi.org/10.26820/reciamuc/5.(4).noviembre.2021.92-99

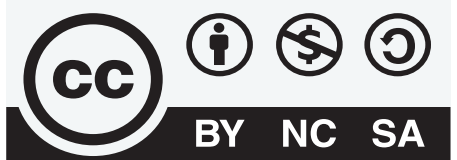

CREATIVE COMMONS RECONOCIMIENTO-NOCOMERCIAL-COMPARTIRIGUAL 4.0. 